\title{
Programmatic implications of the TUMIKIA trial on community-wide treatment for soil-transmitted helminths: further health economic analyses needed before a change in policy
}

Hugo C. Turner ${ }^{1,2^{*}}$ (1) and Donald A. P. Bundy ${ }^{3}$

\begin{abstract}
School-based deworming programmes are currently the main approach used to control the soil-transmitted helminths (STHS). A key unanswered policy question is whether mass drug administration (MDA) should be targeted to the whole community instead, and several trials in this area have been conducted or are currently on-going. A recent well-conducted trial demonstrated that successful community-wide treatment is a feasible strategy for STH control and can be more effective than school-based treatment in reducing prevalence and intensity of hookworm infection. However, we would argue that it is vital that these findings are not taken out of context or over generalised, as the additional health benefits gained from switching to community-wide treatment will vary depending on the STH species and baseline endemicity. Moreover, community-wide treatment will typically be more expensive than schoolbased treatment. The epidemiological evidence for an additional benefit from a switch to community-wide treatment has yet to be proven to represent "good value for money" across different settings. Further work is needed before changes in policy are made regarding the use of community-wide treatment for STH control, including comprehensive assessments of its additional public health benefits and costs across a range of scenarios, accounting for the presence of alternative treatment delivery platforms.
\end{abstract}

Keywords: Soil-transmitted helminths, Policy, Deworming, Community-wide treatment, School-based treatment, Cost

\section{Letter to the Editor}

School-based deworming programmes are currently the main approach used to control the soil-transmitted helminths (STHs) [1]. A key unanswered policy question is whether mass drug administration (MDA) should be targeted to the whole community instead, and several trials in this area have been conducted or are currently

\footnotetext{
*Correspondence: hturner@oucru.org

${ }^{1}$ Oxford University Clinical Research Unit, Wellcome Africa Asia Programme, Ho Chi Minh City, Vietnam

Full list of author information is available at the end of the article
}

on-going [2-4]. For example, Pullan et al. [4] recently published an impressive and comprehensive trial which demonstrated that community-wide treatment of STHs in Kenya is possible and can be more effective for reducing the prevalence and intensity of hookworm (the main helminth in the communities studied). Specifically, they observed that after 24 months and two rounds of treatment, the overall prevalence of hookworm declined from $18.6 \%$ to $13.8 \%$ within the school-based group compared to $17.9 \%$ to $8.0 \%$ within the community-wide treatment group. Their findings also highlighted the equity of the 
community delivery approach and its ability to reach the poorest and most marginalised communities.

An important question arises whether these results support the policy contention that community-wide treatment is cost-effective, and hence should replace school-based treatment as the standard method of delivery for STH control.

In terms of programme effectiveness, there are two key outcomes of interest: additional reductions in morbidity and contribution to breaking transmission. Helminth morbidity is typically correlated to the intensity of infection, and individuals with moderate to heavy intensity infections experience the majority of $\mathrm{STH}$ related morbidity [5-7]. Due to the non-linear relationship between STH prevalence and intensity [8], when the prevalence of infection falls below $20 \%$, the prevalence of these moderate to heavy intensity infections is expected to be relatively low [9]. Due to this, it is likely that the observed additional reductions in prevalence achieved by using community-wide treatment would only lead to small additional gains in averted morbidity. These findings should not be overgeneralised, and the observed benefit may have been partly reduced due to the previous community-wide treatment for lymphatic filariasis that occurred before the study started [4]. In addition, mathematical models have also projected larger benefits of community-wide treatment in settings that have high baseline prevalence of hookworm [10]. However, the additional benefits of community-wide treatment would likely be even lower where Ascaris lumbricoides and Trichuris trichiura are more prevalent, since unlike hookworm, these species typically have much lower levels of infection in adults relative to children [7, 10-12]. Consequently, the additional health benefits gained from switching to community-wide treatment will vary depending on which STH species are endemic, and the baseline level of endemicity [10]. It is likely that in at least some settings both school-based and community-wide approaches can sustain infection below a level likely to be associated with significant morbidity.

A potential further benefit of community-wide treatment for STHs is that it may contribute to breaking transmission [13, 14]. Typically, treatment is followed by re-infection [15], but it is also theorized that sufficient treatment would result in the collapse of transmission (i.e. stochastic extinction), thus removing the need for further MDA. Modelling studies have estimated a breakpoint of around $2 \%$ prevalence for hookworm [16]. However, the reductions in prevalence under community-wide treatment in the TUMIKIA study did not approach this estimated breakpoint within the trial period and the studies available to date in this area have not definitively proven breaking transmission is possible with either school-based or community-wide treatment. The TUMIKIA study also indicates that achieving the projected high coverage and compliance levels estimated to be required to break transmission in some settings in a programmatically realistic time horizon could be difficult within many control programmes.

A major consideration regarding the use of community-wide treatment is its cost [17]. Even when the per treatment costs are lower relative to school-based strategies, the total cost will typically be higher because more individuals are treated. This is illustrated with an example in Table 1. Although this is a crude calculation, it indicates that even with conservative, at-scale estimates, the total cost of community-wide treatment is likely to be more expensive than the total cost of school-based treatment. Does the additional benefit outweigh this larger investment and greater complexity of implementation: is community-based treatment good value for money or cost-effective? Further studies are needed to obtain more accurate estimates of the difference in these costs [17]. There is potential for

Table 1 Hypothetical case study of the estimated financial costs of using different treatment strategies within the Kenyan national STH control programme

\begin{tabular}{llcc}
\hline Strategy & Number treated & Assumed cost per treatment (US\$) & $\begin{array}{c}\text { Estimated total } \\
\text { financial cost per year } \\
\text { (US\$) }\end{array}$ \\
\hline School-based treatment & & $0.30^{\mathrm{b}}-0.56^{\mathrm{c}}$ & $1.8-3.4$ million \\
Community-wide treatment & 6 million children [23] & $0.32^{\mathrm{d}}-0.46^{\mathrm{e}}$ & $4.4-6.4$ million
\end{tabular}

\footnotetext{
a Approximated based on demographic data from the World Bank [24]

b Based on the WHO MDA cost benchmark model [25]

c Estimate from Evidence Action (a programmatic estimate for 2015) [23]

${ }^{d}$ Based on the estimate from the TUMIKIA trial [4]: routine scenario (excluding the research costs) relating to whole county (i.e. estimate at scale). US\$0.025 per treatment was added for the cost of albendazole [4]

e Based on the estimate from the TUMIKIA trial [4]_routine scenario (excluding the research costs) relating to trial areas only. US\$0.025 per treatment was added for the cost of albendazole [4]
} 
community-wide treatment to be cost-saving in the long term if it can stop the need for further treatment [10], but this is contingent on the interruption of transmission in an appropriate timescale, which has yet to be demonstrated.

To conclude, the TUMIKIA study is an important and well-conducted trial which demonstrates that successful community-wide treatment is a feasible strategy for STH control and can be more effective than schoolbased treatment in reducing prevalence and intensity of hookworm infection. However, these results should not be taken out of context, and achieving marginally greater reductions in already low prevalence settings did not necessarily lead to significant public health benefits or break transmission. Yet, community-wide treatment will typically be more expensive than school-based treatment. This does not mean that a community-wide treatment is never cost-effective $[18,19]$, but it does indicate that there are many contexts where switching to communitywide treatment would not yield notable public health benefits compared to remaining with school-based treatment. The policy implications of these results should not be over-interpreted or be extrapolated to all contexts. Ongoing studies (such as DeWorm3) are assessing the capacity of community-wide treatment for breaking STH transmission [3], which could importantly change the policy implications, but this approach currently remains unproven. Importantly, community-wide mass treatment is not the only strategy to provide treatment to adults and it is probable that building on established health system approaches (such as using child health days and antenatal clinics) to deliver treatment could offer a way of treating adults at a lower cost [20,21]. Further work is needed before changes in policy are made regarding the use of community-wide treatment for STH control, including comprehensive assessments of its additional public health benefits and costs across a range of scenarios, accounting for the presence of alternative treatment delivery platforms for reaching at-risk groups other than school-aged children [21, 22].

\section{Abbreviations}

STH: soil-transmitted helminth; MDA: mass drug administration.

\section{Acknowledgements}

Not applicable.

\section{Authors' contributions}

HCT and DAPB conceived the manuscript. HCT wrote the first draft. All of the authors contributed to the writing of the paper. Both authors read and approved the final manuscript.

\section{Funding}

HCT is supported by the Wellcome Trust core Grant (106680/Z/14/Z). DAPB is supported by a grant to the London School of Hygiene and Tropical Medicine from the Children's Investment Fund Foundation.
Availability of data and materials Not applicable.

Ethics approval and consent to participate

Not applicable.

Consent for publication

Not applicable.

\section{Competing interests}

HCT contributed to the TUMIKIA trial. DAPB has worked on the Kenya National Deworming programme with the Government of Kenya, the Kenya Medical Research Institute, the World Bank and the Children's Investment Fund Foundation.

\section{Author details}

1 Oxford University Clinical Research Unit, Wellcome Africa Asia Programme, Ho Chi Minh City, Vietnam. ${ }^{2}$ Centre for Tropical Medicine and Global Health, Nuffield Department of Medicine, University of Oxford, Oxford, UK. ${ }^{3}$ London School of Hygiene and Tropical Medicine, London, UK.

Received: 7 Auqust 2019 Accepted: 17 February 2020

Published online: 27 February 2020

\section{References}

1. WHO. Preventive chemotherapy in human helminthiasis: coordinated use of anthelminthic drugs in control interventions: a manual for health professionals and programme managers. Geneva: World Health Organization; 2006. https://apps.who.int/iris/handle/10665/43545.

2. Clarke NE, Clements ACA, Amaral S, Richardson A, McCarthy JS, McGown J, et al. (S)WASH-D for Worms: a pilot study investigating the differential impact of school- versus community-based integrated control programs for soil-transmitted helminths. PLoS Negl Trop Dis. 2018;12:e0006389.

3. Asbjornsdottir KH, Ajjampur SSR, Anderson RM, Bailey R, Gardiner I, Halliday KE, et al. Assessing the feasibility of interrupting the transmission of soil-transmitted helminths through mass drug administration: the DeWorm3 cluster randomized trial protocol. PLoS Negl Trop Dis 2018:12:e0006166.

4. Pullan RL, Halliday KE, Oswald WE, McHaro C, Beaumont E, Kepha S, et al. Effects, equity, and cost of school-based and community-wide treatment strategies for soil-transmitted helminths in Kenya: a cluster-randomised controlled trial. Lancet. 2019;18(393):2039-50.

5. Chan MS, Medley GF, Jamison D, Bundy DA. The evaluation of potential global morbidity attributable to intestinal nematode infections. Parasitology. 1994;109:373-87.

6. Bundy D, Chan M, Medley G, Jamison D, Savioli L. Chapter 9. Intestinal nematode infections. In: Murray CJL, Lopez AD, Mathers CD, editors. Global epidemiology of infectious disease, vol. IV. Geneva: World Health Organization; 2004

7. Hotez PJ, Bundy DAP, Beegle K, Brooker S, Drake L, de Silva N, et al. Helminth infections: soil-transmitted helminth infections and schistosomiasis. In: Jamison DT, Breman JG, Measham AR, Alleyne G, Claeson M, et al., editors. Disease control priorities in developing countries. 2 nd ed. Washington (DC): World Bank; 2006. p. 467-82.

8. Anderson RM, May RM. Helminth infections of humans: mathematical models, population dynamics, and control. Adv Parasitol. 1985;24:1-101.

9. WHO. Preventive chemotherapy to control soil-transmitted helminth infections in at-risk population groups. Geneva: World Health Organization; 2017. https://www.who.int/nutrition/publications/guidelines/dewor ming/en/.

10. Turner HC, Truscott JE, Bettis AA, Shuford KV, Dunn JC, Hollingsworth TD, et al. An economic evaluation of expanding hookworm control strategies to target the whole community. Parasites Vectors. 2015;8:570.

11. Turner HC, Truscott JE, Hollingsworth TD, Bettis AA, Brooker SJ, Anderson RM. Analysis of the population-level impact of co-administering ivermectin with albendazole or mebendazole for the control and elimination of Trichuris trichiura. Parasite Epidemiol Control. 2016;1:177-87. 
12. Truscott JE, Turner HC, Farrell SH, Anderson RM. Soil-transmitted helminths: mathematical models of transmission, the impact of mass drug administration and transmission elimination criteria. Adv Parasitol. 2016:94:133-98

13. Anderson RM, Truscott JE, Hollingsworth TD. The coverage and frequency of mass drug administration required to eliminate persistent transmission of soil-transmitted helminths. Philos Trans R Soc Lond B Biol Sci. 2014;369:20130435

14. Truscott JE, Hollingsworth TD, Brooker SJ, Anderson RM. Can chemotherapy alone eliminate the transmission of soil transmitted helminths? Parasites Vectors. 2014;7:266.

15. Jia TW, Melville S, Utzinger J, King CH, Zhou XN. Soil-transmitted helminth reinfection after drug treatment: a systematic review and meta-analysis. PLoS Negl Trop Dis. 2012;6:e1621.

16. Truscott JE, Werkman M, Wright JE, Farrell SH, Sarkar R, Asbjornsdottir $\mathrm{K}$, Anderson RM. Identifying optimal threshold statistics for elimination of hookworm using a stochastic simulation model. Parasites Vectors. 2017;10:321.

17. Turner HC, Truscott JE, Hollingsworth TD, Bettis AA, Brooker SJ, Anderson RM. Cost and cost-effectiveness of soil-transmitted helminth treatment programmes: systematic review and research needs. Parasites Vectors. 2015;8:355.

18. Lo NC, Bogoch II, Blackburn BG, Raso G, N'Goran EK, Coulibaly JT, Becker $\mathrm{SL}$, et al. Comparison of community-wide, integrated mass drug administration strategies for schistosomiasis and soil-transmitted helminthiasis: a cost-effectiveness modelling study. Lancet Glob Health. 2015;3:629-38.

19. Lo NC, Lai YS, Karagiannis-Voules DA, Bogoch II, Coulibaly JT, Bendavid E, et al. Assessment of global guidelines for preventive chemotherapy against schistosomiasis and soil-transmitted helminthiasis: a cost-effectiveness modelling study. Lancet Infect Dis. 2016;16:1065-75.

20. Bangert M, Bancalari P, Mupfasoni D, Mikhailov A, Gabrielli AF, Montresor A. Provision of deworming intervention to pregnant women by antenatal services in countries endemic for soil-transmitted helminthiasis. PLoS Negl Trop Dis. 2019;13:e0007406.

21. Chami GF, Bundy DAP. More medicines alone cannot ensure the treatment of neglected tropical diseases. Lancet Infect Dis. 2019;19:e330-6.

22. Keiser J, Utzinger J. Community-wide soil-transmitted helminth treatment is equity-effective. Lancet. 2019;393:2011-2.

23. Evidence Action. 2016 Costs of school-based deworming: a best-buy development intervention; 2017. https://www.evidenceaction.org/blogfull/deworm-cost-per-child.

24. World Bank: World Development Indicators. https://data.worldbank.org/ indicator.

25. Fitzpatrick C, Fleming FM, Madin-Warburton M, Schneider T, Meheus F, Asiedu K, et al. Benchmarking the cost per person of mass treatment for selected neglected tropical diseases: an approach based on literature review and meta-regression with web-based software application. PLoS Negl Trop Dis. 2016;10:e0005037.

\section{Publisher's Note}

Springer Nature remains neutral with regard to jurisdictional claims in published maps and institutional affiliations.
Ready to submit your research? Choose BMC and benefit from:

- fast, convenient online submission

- thorough peer review by experienced researchers in your field

- rapid publication on acceptance

- support for research data, including large and complex data types

- gold Open Access which fosters wider collaboration and increased citations

- maximum visibility for your research: over $100 \mathrm{M}$ website views per year

At $\mathrm{BMC}$, research is always in progress.

Learn more biomedcentral.com/submissions 